\title{
Concept of Blood Diagnosis Bio-Chip
}

\author{
S. P. Wadkar ${ }^{*}$, Shubham S. Mahajan ${ }^{\dagger}$, Anagha P. Joshi ${ }^{\dagger}$ and Neeraj Shende" \\ †Department of Mechanical Engineering, MIT College of Engineering, Kothrud, Pune, Maharashtra 411038, India \\ \#Serum Institute of India Pvt. Ltd, India
}

Accepted 02 March 2016, Available online 15 March 2016, Special Issue-4 (March 2016)

\begin{abstract}
Much research has not been done in the field of Microfluidics yet because working in this field means working in the range of tens to hundreds of micrometer that requires much precise handling of the volume of the fluid. There is an essential need for the rapid diagnosis of the blood for detecting the various vital diseases affecting the human body. These diseases, if not diagnosed properly would reach to a vital stage, which may deplete the physical and mental condition of the human body and even cause death. There are many methods developed for the blood diagnosis but these conventional methods which are available require more time for the detection of the diseases and also these tests become cumbersome due to a large number of equipment required for the same. An idea is to be developed a device which would help in plasma or serum separation from the blood and also diagnose the blood in a shorter time than the conventional methods which would be inexpensive and portable. This lab-on-chip device focuses mostly on the principle and development of microfluidics.
\end{abstract}

Keywords: Microfluidics, lab-on-chip, plasma separation

\section{Introduction}

Every minute, the human body's entire blood volume is recirculated; as a result, blood is a treasure-trove of information about the functioning of the body, particularly at the molecular level. A major milestone in microfluidics could soon lead to stand-alone, selfpowered chips that can diagnose diseases within minutes. The dream of a true lab-on-a-chip has been around for a while, but most systems developed thus far have not been truly autonomous, said Ivan Dimov, UC Berkeley post-doctoral researcher in bioengineering and co-lead author of the study. Dimov works in the lab of the study's principal investigator, Luke Lee, UC Berkeley professor of bioengineering and co-director of the Berkeley Sensor and Actuator Center. This is a very important development for global healthcare diagnostics, said Lee. Field workers would be able to use this device to detect diseases such as HIV or tuberculosis in a matter of minutes. The fact that we reduced the complexity of the biochip and used plastic components makes it much easier to manufacture in high volume at low cost. Our goal is to address global health care needs with diagnostic devices that are functional, cheap and truly portable.

The biochip uses trenches patterned underneath microfluidic channels that are about the width of a human hair. When whole blood is dropped onto the chip's inlets, the relatively heavy red and white blood

*Corresponding author: S. P. Wadkar cells settle down into the trenches, separating from the clear blood plasma. The blood moves through the chip in a process called degas-driven flow.

Here the blood merely two drops of $10 \mathrm{~mm}$ diameter each are dropped in the portion shown on the chip. This blood flows to the trench. Here plasma and $\mathrm{RBC}, \mathrm{WBC}$ are separated by the density difference. RBC and WBC being heavier settles down and plasma being lighter floats which used for further testing. The primary objective is to replace laborious sample preparation steps by integrating blood plasma separation and multiplexed assays on the same device. In other techniques plasma is separated by rotating the bottle containing blood at a certain speed to produce the centrifugal effect. This causes the plasma to be accumulated at the centre of rotation. Therefore it makes the process costlier because of costlier equipment to separate plasma. Separated plasma from the trench flows through the flow channel, this flow channel causes the flow of the plasma below the surface which is coated with the proteins which react with the antibodies in the plasma. This reaction either causes some colour change orfluorescence effect over this surface which can be observed on this protein coated surface. The flow required here is obtained by degas driven flow. Here the suction device is like sponge. This device is placed in vacuum so that the air in it is taken out and when this device is connected to the flow channel it causes the flow because of suction effect. For the biochip, proper reaction should occur for 
which the flow in the rectangular trench of the biochip should remain there for the time equal to the reaction time between the antibodies and the proteins coated. There should be sufficient turbulence inside the trench which is difficult to attain when it comes to microfluidics because the turbulence will ensure that almost all the antibodies will react with the proteins. The another objective is to make the fluid move effectively over the area of the protein coated surface to ensure proper reaction.

\section{The Concept Model}

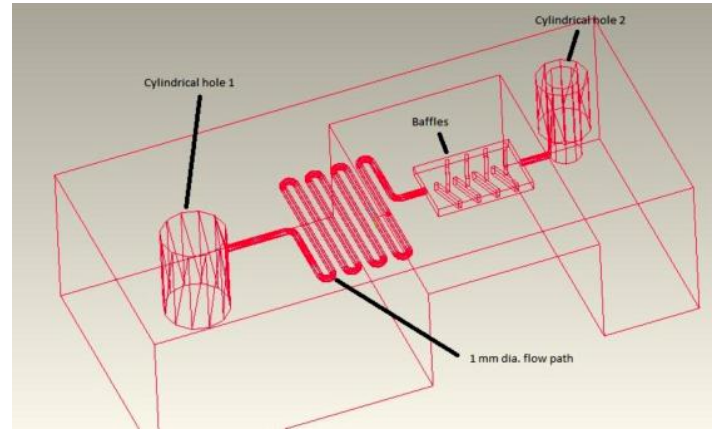

Fig.2(a) The concept model developed using CAD software

While modelling, flow diameter was selected as $1 \mathrm{~mm}$. One blood drop of size $14 \mathrm{~mm}$ diameter is dropped in the cylindrical hole 1 where plasma will be separated by density difference. This plasma will flow through the flow channels as shown in fig., the flow channels are given certain number of turns. The number of turns will be decided based upon the velocity of the fluid required at the inlet of the rectangular trench where baffles are there to ensure that proper turbulence will be created and the fluid will move over all the area of the trench. After the fluid has moved through the rectangular trench then it passes to cylindrical hole 2 where tube and syringe is attached to suck out the fluid. Below the rectangular trench the protein coated paper is attached with the help of a block.

For the flow to occur, a pressure differential at the two ends of the flow on the biochip is preferred. Therefore, the boundary conditions are selected as;

For inlet, pressure $=1.01325$ bar (absolute)

For outlet, pressure $=0.9$ bar (absolute)

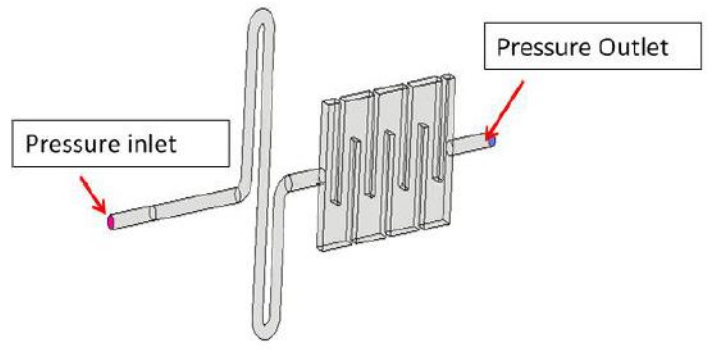

Fig. 2(b) Model shows pressure differential at the two ends
Detailed drawing of the main part:

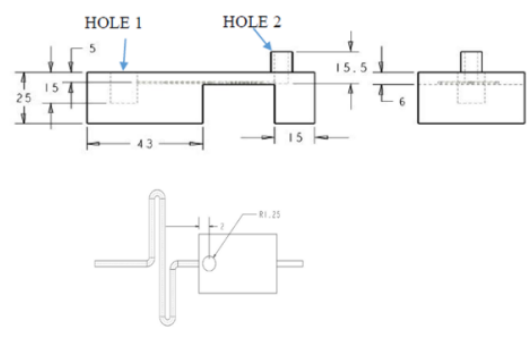

Fig. 2(c): Detailed drawing of the model

The total length of the biochip is approximately equal to $85 \mathrm{~mm}$

\section{Material and Methods}

The material which can be used for the fabrication of the biochip are PDMS (polymethyldisiloxane), ABS plastic, acrylic glass, etc which have quite similar properties. The material must be selected in such a way that it must not react with blood so as not to affect the results. Such a biochip can be manufactured by the rapid prototyping processes such as 3D printing, stereolithography.

\section{Manufactured model}

The cost of manufacturing a single model by 3D printing is much higher. It would be less if a large number of models are manufactured by the same method. Therefore the prototype for this purpose was manufactured at a scale of 1:3. The prototype was manufactured by glass blowing fabrication method For this, a glass tube was selected of $3 \mathrm{~mm}$ inner diameter and $5 \mathrm{~mm}$ outer diameter. The part is made up of borosilicate glass because of its property not to react with blood. The rectangular trench was made up of acrylic material separately and then assembled with the help of a glue.

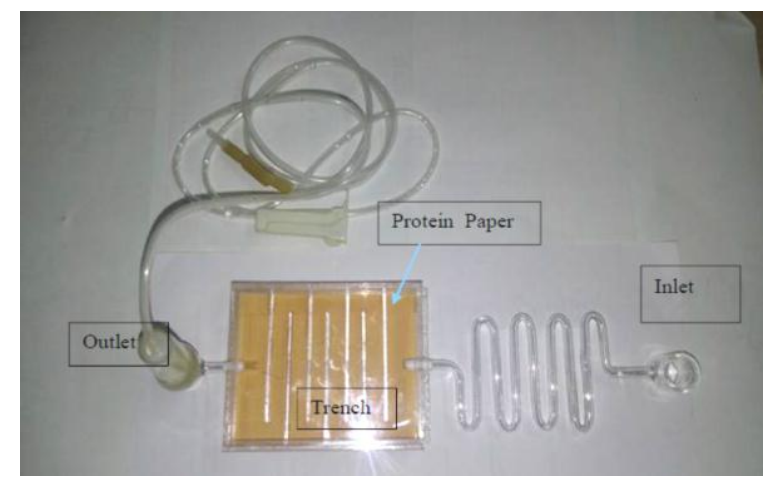

Fig.4 The prototype manufactured to a scale of 1:3

\section{Conclusions}

The concept of biochip for the blood diagnosis of the diseases can be used to develop a bio-chip for the rapid blood diagnosis. The turns are provided in the model to 
enhance the reaction of the biomarkers with the blood and also to increase the reaction time. The bio-chip is more reliable than the conventional methods as the reaction time is reduced and if the production is large, the cost reduces to a large extent. Also the bio-chip is of small size which makes it portable.

\section{References}

IvanK.Dimov, Antonio J.Ricco Self Powered Integrated Microfluidic Blood Analysis System (SIMBAS)-; 13th International Conference On MiniaturizedSystemsNov 1-5 , 2009.
David Y Liang, Luke P.Lee Systematic Characterization Of Degas Driven Flow For PDMS MicrofluidicDevicesBiomicrofluidics 2 June, 2011.

Ali Asgar, Chwee Teek Lim Microfluidics For Cell SeparationInternationalFederation For Medical And Biological Engineering , 2010.

Wie Cheng Tian And ErinFinehout,.Microfluidics For Biological Applications, Springer.

Suman Chakraborty,Microfluidics \& Microfabrication, Springer

IvanK.Dimov,Luke P.Lee ,Antonio J.Ricco Self Contained, Self Integrated Microfluidic Blood Analysis System; Royal Society of Chemistry 2010 